\title{
openheart Value of FEops HEARTguide patient- specific computational simulations in the planning of left atrial appendage closure with the Amplatzer Amulet closure device: rationale and design of the PREDICT-LAA study
}

\begin{abstract}
Philippe Garot, ${ }^{1}$ Xavier Iriart, ${ }^{2}$ Adel Aminian, ${ }^{3}$ Joelle Kefer, ${ }^{4}$ Xavier Freixa, ${ }^{5}$ Ignacio Cruz-Gonzalez, ${ }^{6}$ Sergio Berti, ${ }^{7}$ Liesbeth Rosseel, ${ }^{8}$ Reda Ibrahim, ${ }^{9}$ Kasper Korsholm, ${ }^{10}$ Jacob Odenstedt, ${ }^{11}$ Jens-Erik Nielsen-Kudsk, ${ }^{10}$ Jaqueline Saw, ${ }^{12}$ Lars Sondergaard, ${ }^{13}$ Ole De Backer (D) ${ }^{13}$
\end{abstract}

\section{- Additional material is published online only. To view please visit the journal online (http://dx.doi.org/10.1136/ openhrt-2020-001326).}

To cite: Garot $P$, Iriart $X$, Aminian $A$, et al. Value of FEops HEARTguide patient-specific computational simulations in the planning of left atrial appendage closure with the Amplatzer Amulet closure device: rationale and design of the PREDICT-LAA study. Open Heart 2020;7:e001326. doi:10.1136/ openhrt-2020-001326

Received 28 April 2020 Revised 26 May 2020 Accepted 27 May 2020
Check for updates

\section{C) Author(s) (or their} employer(s)) 2020. Re-use permitted under CC BY-NC. No commercial re-use. See rights and permissions. Published by BMJ.

For numbered affiliations see end of article.

Correspondence to Dr Ole De Backer; ole. debacker@gmail.com

\section{ABSTRACT}

Background 0ptimal preprocedural planning is essential to ensure successful device closure of the left atrial appendage (LAA).

Design The PREDICT-LAA study is a prospective, international, multicentre, randomised controlled trial ( ClinicalTrials.gov NCT04180605). Two hundred patients eligible for LAA closure with an Amplatzer Amulet device (Abbott, USA) will be enrolled in the study. Patients will be allocated to a computational simulation arm (experimental) or standard treatment arm (control) using a 1:1 randomisation. For patients randomised to the computational simulation arm, preprocedural planning will be based on the analysis of cardiac computed tomography (CCT)-based patient-specific computational simulations (FEops HEARTguide, Ghent, Belgium) in order to predict optimal device size and position. For patients in the control arm, preprocedural planning will be based on local practice including CCT analysis. The LAA closure procedure and postprocedural antithrombotic therapy will follow local practice in both arms. The primary endpoint of the study is incomplete LAA closure and device-related thrombus as assessed at 3 months postprocedural CCT. Secondary endpoints encompass procedural efficiency (number of devices used, number of repositioning, procedural time, radiation exposure, contrast dye), procedure-related complications within 7 days postprocedure and a composite of all-cause death and thromboembolic events at 12 months.

Conclusion The objective of the PREDICT-LAA study is to test the hypothesis that a preprocedural planning for LAA closure with the Amplatzer Amulet device based on patient-specific computational simulations can result in a more efficient procedure, optimised procedural outcomes and better clinical outcomes as compared with a standard preprocedural planning.

Trial registration number ClinicalTrials.gov Registry (NCT04180605).

\section{BACKGROUND}

Percutaneous left atrial appendage (LAA) closure is being increasingly used as a treatment strategy to prevent stroke in patients with non-valvular atrial fibrillation (NVAF) and contraindication(s) to oral anticoagulant therapy. In order to obtain a successful LAA closure, correct LAA closure device size selection as well as optimal implantation should be pursued. ${ }^{1-5}$

Various cardiac imaging techniques are currently used to assess the anatomy and size of the LAA, ranging from two-dimensional transoesophageal echocardiographic (TEE) to cardiac computed tomography (CCT), which allows three-dimensional (3D) evaluation of the LAA and its surrounding structures. Although more detailed preprocedural LAA imaging by CCT helps to better understand and size the patient's LAA anatomy, ${ }^{3}$ predicting the actual 'landing zone' of the LAA closure device still remains difficult. The use of printed 3D-LAA models has been reported as a method to improve the preprocedural planning ${ }^{45}$; however, this approach is not feasible in all cases due to the logistics required with 3D printing.

The use of CCT-based patient-specific computational models to virtually deploy the closure device into the reconstructed patient-specific LAA anatomy is a more versatile alternative to bench testing in $3 \mathrm{D}$ printed models and provides additional information to cardiac imaging techniques only. The patient-specific computational simulation aims to reproduce the mechanical interaction 
between device and the patient's anatomy and provides a deeper insight into the behaviour of the LAA closure device before the procedure. The FEops HEARTguide simulation technology (FEops NV, Ghent, Belgium) has been validated for LAA closure ${ }^{6}$ as well as for transcatheter aortic valve replacement procedures. ${ }^{78}$

The PREDICT-LAA clinical study investigates the hypothesis that a better preprocedural planning can be achieved by consulting FEops HEARTguide, simulating different LAA closure device sizes and positions in a patient-specific LAA anatomy and thereby providing the implanter an overview of possible optimal and suboptimal scenarios. This better preprocedural planning may result in higher rates of complete LAA closure with lower rates of DRT as assessed on postprocedural CCT imaging.

\section{METHODS}

\section{Study objectives}

The purpose of this trial is to study the possible added value of FEops HEARTguide patient-specific computational simulations in the preprocedural planning of percutaneous LAA closure with the Amplatzer Amulet device, with focus on procedural safety and efficiency as well as on clinical outcomes.

\section{Study population and patient selection}

Two hundred patients with NVAF and eligible for LAA closure with an Amplatzer Amulet LAA closure device will be enrolled at up to 12 European and Canadian sites. Only patients referred to and approved for percutaneous LAA closure-according to local practice and legislation-can be considered for enrolment in the trial. All patients should be 18 years or older and should sign a written informed consent. Key exclusion criteria are a reduced renal function (with glomerular filtration rate $<30 \mathrm{~mL} / \mathrm{min} / 1.73 \mathrm{~m}^{3}$ ), iodine contrast allergy or any other condition that prohibits CCT imaging, and suboptimal quality of the preprocedural CCT scan.

\section{Randomisation and treatment protocol}

Consenting subjects will be randomised in a 1:1 ratio to a standard treatment arm or computational simulation arm. Randomisation will be performed through a secure web-based REDCap application at the time of inclusion of the patient in the trial. The randomisation will be stratified by site. The study design is shown in figure 1. Patients randomised to the standard treatment arm will be treated according to the site's routine practice-as preprocedural imaging, a CCT scan has to be performed; this can also be complemented with TEE at the discretion of the operator. For patients randomised to the computational simulation arm, the planning of the LAA closure will be performed according to the standard practice of the site integrated with a careful review of the FEops HEARTguide simulation results. The only prerequisite is that the preprocedural CCT scan has to be uploaded into the FEops HEARTguide platform in a pseudo-anonymised fashion. The results of the computational simulation will be provided to the operator within two working days, containing a range of options in terms of device size and implant position in the selected patient (figure 2). The operator should use the computational simulations as an additional preprocedural planning tool, aiming for a $10 \%$ to $25 \%$ compression of the Amulet lobe, complete LAA closure with full coverage of all trabeculations and a concave-shaped disc that is not retracted into the LAA neck (online supplementary video 1 ). The preprocedural planning should be performed integrating the standard planning with the analysis of the patient-specific computational results. For all patients, the LAA closure procedure should be performed following the routine practice of the site with TEE, micro-TEE or intracardiac echocardiography guidance, either in general or local anaesthesia. All patients should receive postprocedural antithrombotic medical therapy according to the participating sites' routine practice, which is at the discretion of the treating physician. All patients enrolled in the study should have a postprocedural CCT scan at 3 months after the procedure, to assess for complete LAA closure, DRT

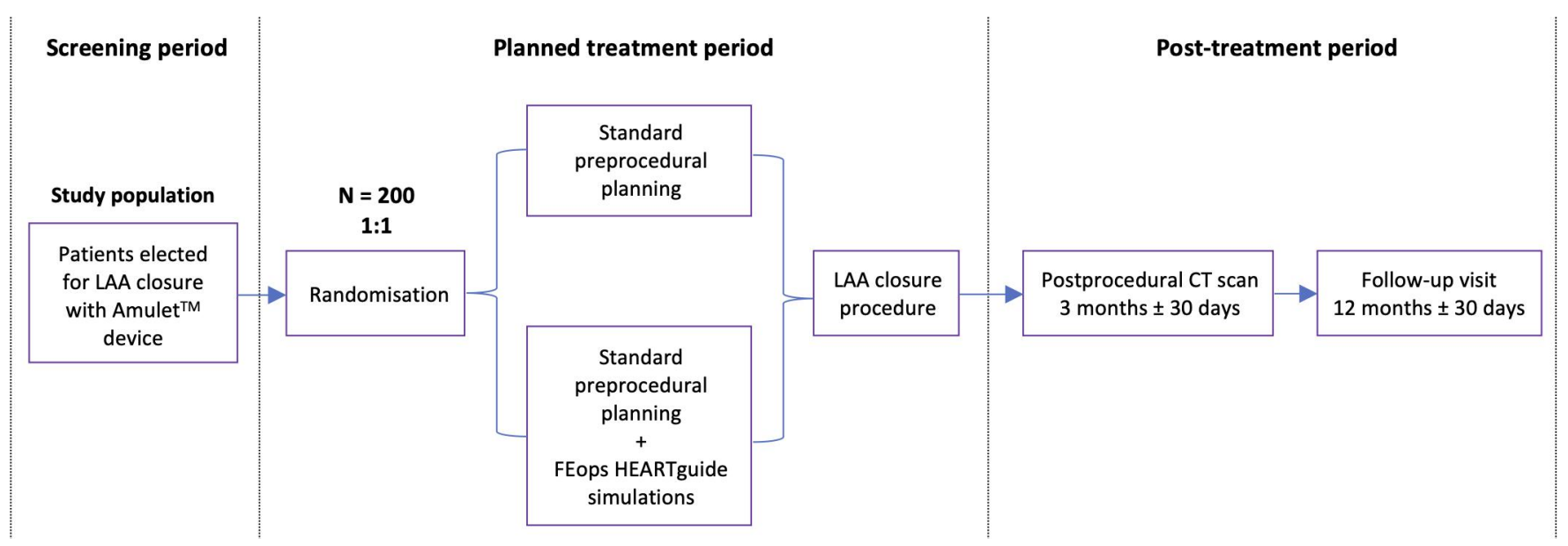

Figure 1 PREDICT-LAA study workflow. LAA, left atrial appendage. 

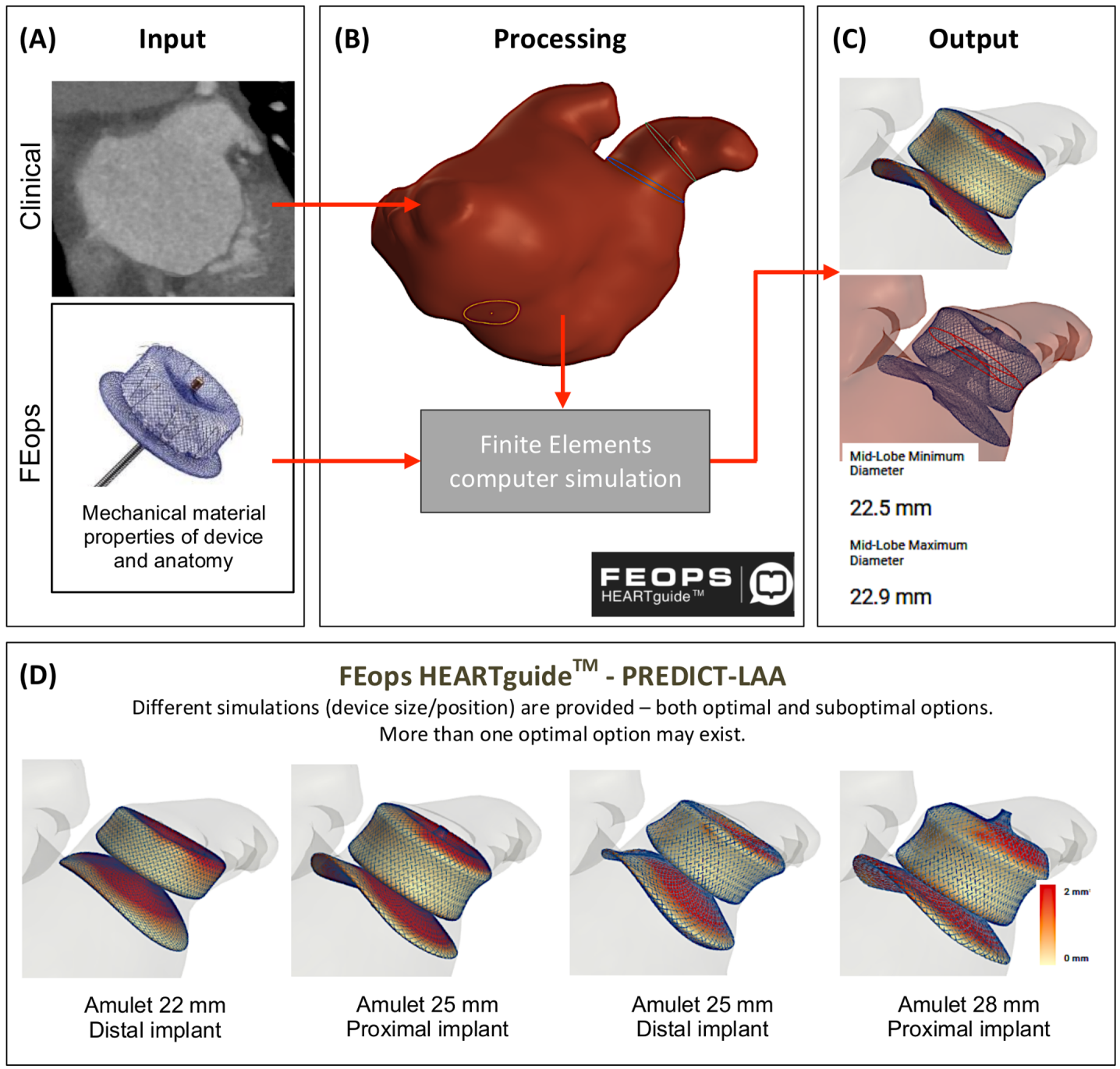

Figure 2 FEops HEARTguide workflow and simulations. (Panel A) The preprocedural CT scan of the selected patient is uploaded on the web-based platform FEops HEARTguide and is-together with the in-house computational model of the Amulet device with adequate material properties for device and anatomy-used as input to the workflow. (Panel B) The received images are further processed to extract three-dimensional patient-specific anatomical reconstructions and landmarks for the procedure. This, in combination with the device model, serves as input for the computational finite element analysis. (Panel C) As output, several options in terms of device size and position are provided, including LAA wall apposition plots (colour-scale indicating the distance between the device and the anatomy), deformation visualisation and measurements (at the section indicated in red in panel C). (Panel D) The use of the simulation output in clinical practice: for one single patient, different simulations in terms of device size and position are provided to the operator, who can gain additional insights on the device-host interaction before the procedure.

and device position. All patients enrolled in the study should also have a clinical follow-up visit at 12 months after the procedure to record possible thromboembolic events or all-cause mortality (table 1A). The list of medical investigations required is shown in table $1 \mathrm{~B}$.

\section{Study endpoints}

The primary endpoint of the PREDICT-LAA study is the percentage of patients with incomplete LAA closure (defined as any remaining contrast leakage into the LAA distal of the Amulet lobe) and/or a definite DRT at postprocedural CCT imaging at 3 months after the procedure. Definite DRT is defined as 'high-grade' hypoattenuating thickening at the atrial surface of the closure deviceas previously described by Korsholm et al. ${ }^{9}$ Secondary endpoints evaluated in this study are listed in box 1 . The CCT CoreLab evaluation will be performed at Rigshospitalet, Copenhagen, Denmark ${ }^{9}$; the readers of the CCT scan will be blinded from the baseline patient and procedural data as well as from patient's randomisation arm.

\section{Sample size calculation}

The primary endpoint is expected to occur in $30 \%$ of patients in the standard treatment arm. An equal number of patients will be enrolled in both treatment arms. A $65 \%$ reduction of the primary endpoint requires inclusion of 174 patients in order to demonstrate superiority (power $0.8, \alpha$ $0.05)$. Taking into account an estimated loss at follow-up of $10 \%$ to $15 \%$ of patients (due to mortality and inconclusive CCT imaging at 3 months postprocedure), the total sample 
Table 1A Schedule for patients enrolled in PREDICT-LAA study

\begin{tabular}{|c|c|c|c|}
\hline & Standard treatment arm & \multicolumn{2}{|c|}{ Computational simulation arm } \\
\hline Preprocedural CT scan & Standard & \multicolumn{2}{|c|}{ Upload images to FEops HEARTguide } \\
\hline Preprocedural planning & Standard & \multicolumn{2}{|c|}{ Standard+FEops HEARTguide results } \\
\hline LAA closure procedure & Standard & \multicolumn{2}{|l|}{ Standard } \\
\hline Antithrombotic therapy & Standard & \multicolumn{2}{|l|}{ Standard } \\
\hline Postprocedural CT scan & At 3 months \pm 30 days & \multicolumn{2}{|c|}{ At 3 months \pm 30 days } \\
\hline Follow-up visit & At 12 month \pm 30 days & \multicolumn{2}{|c|}{ At 12 months \pm 30 days } \\
\hline & Preprocedure & At 3 months & At 12 months \\
\hline Routine medical check & Yes & Yes & Yes \\
\hline 12-Lead ECG & Yes & Yes & Yes \\
\hline Transthoracic echocardiography & Yes & Yes & No \\
\hline Cardiac CT scan & Yes & Yes & No \\
\hline
\end{tabular}

size needed to demonstrate superiority has been calculated to be 200 patients. The primary endpoint will be analysed using Fisher's exact test or a $\chi^{2}$ test, as required. Data/statistical analysis will be performed according to the 'intentionto-treat' principle as a first approach and 'as-treated' as a second approach.

\section{Study organisation}

PREDICT-LAA is an investigator-initiated, prospective, international, randomised clinical trial that will be executed under the academic leadership of investigators at Rigshospitalet University Hospital, Copenhagen

\section{Box 1 PREDICT-LAA study endpoints}

Primary endpoint

- Incomplete closure of the LAA with remaining contrast leakage into the LAA distal of the Amulet lobe and/or presence of a definite device-related thrombus as assessed at postprocedural CCT at 3 months after LAA closure.

Secondary endpoints

- Number of LAA closure devices used per procedure.

- Number of LAA closure device repositionings per procedure. Repositioning is defined as full deployment of the Amulet lobe in the LAA, followed by either full or partial recapture and redeployment of the lobe.

- Procedural time, radiation exposure and amount of contrast medium used per procedure.

- Procedure-related complications encompassing device embolisation, pericardial effusion requiring intervention, procedure-related stroke and procedure-related death within 7 days of the procedure.

- Different degrees of contrast leakage into the LAA.

- Full coverage of all LAA trabeculations by the Amulet device and a concave-shaped disc without retraction of the disc into the LAA neck as assessed at postprocedural CCT scan.

- Composite of all-cause death and thromboembolic event (transient ischaemic attack, ischaemic stroke or systemic embolism) at 12 months after randomisation.

CCT, cardiac computed tomography; LAA, left atrial appendage.
(Denmark) and Institut Cardiovasculaire Paris Sud, Massy, Paris (France). The study will be conducted at up to 12 European and Canadian sites in compliance with the Declaration of Helsinki, International Conference on Harmonization, Good Clinical Practice Guidelines and applicable regulatory requirements. The first patient was enrolled in the study on 14 January 2020 and enrolment of all 200 patients is expected being completed in December 2021 with the last patient coming for the 1 year follow-up visit in December 2022. Five patients were enrolled as of the date of manuscript submission. The final study protocol and informed consent have been reviewed and approved by the local ethics boards, institutional review boards and corresponding health authorities of all participating sites. Rigshospitalet takes the sponsor role in this clinical trial. The study is funded by Abbott (Minneapolis, Minnesota, USA) and FEops NV (Ghent, Belgium). The academic sponsor will have full access to the trial data and will submit the results for publication in a peer-reviewed medical journal.

\section{CONCLUSION}

The PREDICT-LAA clinical trial is the first randomised clinical trial studying the efficacy of the preprocedural planning for percutaneous LAA closure, comparing a standard approach with a preprocedural planning that integrates patient-specific computational simulations. Hence, the PREDICT-LAA study will provide, as a first, randomised data on the possible added value of patientspecific computational simulations in the preprocedural planning for LAA closure.

\section{Author affiliations}

${ }^{1}$ Department of Cardiology, Institut Cardiovasculaire Paris Sud, Massy, Île-deFrance, France

${ }^{2}$ Pediatric and Congenital Cardiology, University Hospital of Bordeaux, Pessac, MS, France

${ }^{3}$ Department of Cardiology, Centre Hospitalier Universitaire de Charleroi, Charleroi, Hainaut, Belgium 
${ }^{4}$ Division of Cardiology, Cliniques Universitaires Saint-Luc, Brussels, Belgium ${ }^{5}$ Cardiovascular Institute, Hospital Clinic de Barcelona, Barcelona, Catalunya, Spain ${ }^{6}$ Department of Cardiology, Hospital Clínico Universitario de Salamanca, Salamanca, Spain

${ }^{7}$ Cardiology Unit, Fondazione CNR Regione Toscana, Massa, Italy

${ }^{8}$ Department of Cardiology, University Hospital Galway, Galway, Ireland

${ }^{9}$ Department of Cardiology, Montreal Heart Institute, Montreal, Quebec, Canada

${ }^{10}$ Department of Cardiology, Aarhus Universitetshospital Skejby, Aarhus, Denmark

${ }^{11}$ Department of Cardiology, Sahlgrenska University Hospital, Goteborg, Sweden

${ }^{12}$ Department of Cardiology, Vancouver General Hospital, Vancouver, British

Columbia, Canada

${ }^{13}$ Department of Cardiology, Rigshospitalet, Copenhagen, Denmark

Contributors $\mathrm{PG}$ and $\mathrm{ODB}$ designed the study and prepared the first draft of the manuscript. XI, AA, JK, XF, ICG, SB, LR, RI, KK, JO, JENK, JS and LS contributed to manuscript revision. All authors will contribute to enrolment and patient treatment in the study.

Funding The study is funded by Abbott (Minneapolis, MN, USA) and FEops NV (Ghent, Belgium).

Competing interests $\mathrm{ODB}$ and LS have received institutional research grants from Abbott and FEops NV.

Patient consent for publication Not required.

Provenance and peer review Not commissioned; externally peer reviewed.

Data availability statement Data may be obtained from a third party and are not publicly available. This is a study protocol, not containing study results/data yet.

Open access This is an open access article distributed in accordance with the Creative Commons Attribution Non Commercial (CC BY-NC 4.0) license, which permits others to distribute, remix, adapt, build upon this work non-commercially, and license their derivative works on different terms, provided the original work is properly cited, appropriate credit is given, any changes made indicated, and the use is non-commercial. See: http://creativecommons.org/licenses/by-nc/4.0/.
ORCID iD

Ole De Backer http://orcid.org/0000-0002-9674-0278

\section{REFERENCES}

1 Reddy VY, Sievert H, Halperin J, et al. Percutaneous left atrial appendage closure vs warfarin for atrial fibrillation: a randomized clinical trial. JAMA 2014;312:1988-98.

2 De Backer O, Arnous S, Ihlemann N, et al. Percutaneous left atrial appendage occlusion for stroke prevention in atrial fibrillation: an update. Open Heart 2014;1:e000020.

3 Korsholm K, Berti S, Iriart X, et al. Expert Recommendations on Cardiac Computed Tomography for Planning Transcatheter Left Atrial Appendage Occlusion. JACC Cardiovasc Interv 2020;13:277-92.

4 Otton JM, Spina R, Sulas R, et al. Left atrial appendage closure guided by personalized 3D-printed cardiac reconstruction. JACC Cardiovasc Interv 2015;8:1004-6.

5 Ciobotaru V, Combes N, Martin CA, et al. Left atrial appendage occlusion simulation based on three-dimensional printing: new insights into outcome and technique. Eurolntervention 2018;14:176-84.

6 Bavo AM, Wilkins BT, Garot P, et al. Validation of a computational model aiming to optimize preprocedural planning in percutaneous left atrial appendage closure. $J$ Cardiovasc Comput Tomogr 2020;14:149-54.

7 de Jaegere P, Rocatello G, Prendergast BD, et al. Patient-Specific computer simulation for transcatheter cardiac interventions: what a clinician needs to know. Heart 2019;105:s21-7.

8 Rocatello G, El Faquir N, De Santis G, et al. Patient-Specific computer simulation to elucidate the role of contact pressure in the development of new conduction abnormalities after Catheter-Based implantation of a self-expanding aortic valve. Circ Cardiovasc Interv 2018;11:e005344.

9 Korsholm K, Jensen JM, Nørgaard BL, et al. Detection of devicerelated thrombosis following left atrial appendage occlusion: a comparison between cardiac computed tomography and transesophageal echocardiography. Circ Cardiovasc Interv 2019;12:e008112. 